\title{
CARACTERÍSTICAS DE LA ESCRITURA DE NIÑOS EN SITUACIÓN DE CONTACTO GUARANÍ-ESPAÑOL EN ESPACIOS URBANOS: UNA PROPUESTA DE INVESTIGACIÓN
}

\author{
CHARACTERISTICS OF THE WRITING OF CHILDREN IN A GUARANÍ- \\ SPANISH CONTACT SITUATION IN URBAN SPACES: A RESEARCH PROPOSAL
}

Carolina Ayala ${ }^{1}$

ayala.carolina@gmail.com

\begin{abstract}
Resumen
En este artículo se presentan aspectos del diseño de una investigación en curso, centrada en las características de la escritura de niños de tercero a quinto año de Educación Primaria en contextos urbanos, que se encuentran en situación de contacto guaraní-español. La perspectiva teórica y metodológica adoptada es la de la Etnopragmática (García, 1995). Nuestra hipótesis inicial es que las elecciones que realicen los sujetos en situación de contacto lingüístico presentan diferencias con los monolingües en español y con la variedad estandarizada del español, en función de conceptualizaciones cognitivas que responden a aspectos culturales y a las características del guaraní en tanto lengua de sustrato (García, 1995; Martínez, 2000, 2003, 2009, 2010b, 2010c, 2013, 2015a; Martínez y Speranza, 2009a; Martínez, Speranza y Fernández, 2006a; Speranza, 2010, 2014). Puesto que se trata de una investigación en curso, de la que aún no se han obtenido resultados, se desarrollarán aspectos relativos a la selección y justificación del eje de la investigación; asimismo, se presentarán aspectos centrales del diseño metodológico y del marco teórico. Por último, se presentarán aspectos referidos al tipo de conocimiento que se pretende generar con este trabajo y su importancia para posteriores aportes para la intervención didáctica en alfabetización focalizadas en el contacto de lenguas.
\end{abstract}

Palabras clave: Contacto lingüístico, Guaraní, Español, Etnopragmática, Educación 


\begin{abstract}
This article presents comments on the design of ongoing research focusing onwriting by children in third to fifth grade of Elementary Education, in Guaraní-Spanish contact settings, and in urban contexts.. The theoretical framework adopted is Ethnopragmatics (García, 1995). The initial hypothesis is that the linguistic choices made by subjects in language contact settings are different from those of Spanish monolinguals. I also hypothesize that differences across groups are based on cognitive conceptualizations that respond to cultural aspects and features of Guaraní as a substrate language (García, 1995; Martínez, 2000, 2003, 2009, 2010b, 2010c, 2013, 2015a; Martínez y Speranza, 2009a; Martínez, Speranza y Fernández, 2006a; Speranza, $2010,2014)$. Since this is an ongoing investigation, for which results have not yet been obtained, The present article motivates this research, as well as methodological choices and a theoretical background. Finally, I discuss the type of knowledge the present article aims to create, as well as the relevance for future contributions for pedagogical interventions infor literacy in language contact settings.
\end{abstract}

Keywords: Linguistic contact, Guaraní, Español, Ethnopragmatics, Education 


\section{INTRODUCCIÓN}

La realidad sociolingüística de nuestro país muestra que, lejos de ser un país monolingüe en español -tal como se proclamó desde el siglo XIX y durante gran parte del siglo XX-, Argentina es un país multilingüe y multicultural, tanto por la presencia de lenguas de pueblos originarios como por la presencia de lenguas de origen indoamericano que son habladas por población 'criolla': tal es el caso del guaraní (Censabella, 1999). En Argentina, la presencia de las lenguas de la familia tupí guaraní se da focalizada en algunos lugares de población originaria (mbÿa guaraní en Misiones; avá guaraní en Jujuy y Salta; tapiete en Salta, principalmente en Tartagal) y en muchos lugares en los que población criolla habla la lengua (Misiones, Corrientes, Chaco, Formosa). Los grandes centros urbanos, como el Gran Rosario, Córdoba y el Área Metropolitana de Buenos Aires (AMBA) son lugares en donde se asienta mucha población proveniente de Paraguay, así como también de provincias del litoral y de la región chaqueña de nuestro país.

Por supuesto, esa presencia se encuentra también en las aulas de muchas de las escuelas de las regiones mencionadas. Esto propone desafíos y, en la práctica concreta, dificultades para las/os docentes, quienes se encuentran muchas veces con niños y niñas que son monolingües en guaraní, bilingües guaraní-español o aparentemente monolingües en español pero que manejan una variedad del español distinta a la estandarizada, producto del contacto centenario entre las lenguas. Se generan, por parte de los y las docentes, dos posibilidades: el no reconocimiento (no intencional) del bilingüismo y de las variedades; o su reconocimiento como un problema que interfiere, inevitablemente, en el aprendizaje del español. En uno u otro caso se trabaja con estos niños del mismo modo que con monolingües en español, lo que lleva, innumerable cantidad de veces, a su fracaso escolar debido a las dificultades que se presentan para aprender el español estándar no solo en la lengua oral sino -fundamentalmente- en relación con la escritura.

La Provincia de Buenos Aires (así como muchos otros centros urbanos) no tiene contemplada en la formación docente inicial para el Nivel Primario contenidos sobre interculturalidad, bilingüismo ni diversidad lingüística; tampoco existen programas específicos para atender a las necesidades de población educativa que presenta distintos grados de bilingüismo y/o contacto lingüístico con lenguas no indoeuropeas. De ese modo, las prácticas en torno a la enseñanza del castellano se realizan sin atender a las particularidades que presenta la diversidad señalada (que, muchas veces, tampoco está reconocida). Ante esta situación, nos planteamos una investigación que pueda generar conocimiento, fundamentalmente, para la comprensión de los fenómenos de contacto lingüístico en la escuela primaria.

El presente artículo parte de una investigación en curso sobre el contacto guaraníespañol en escrituras de niños/as de tercero a quinto año de la Educación Primaria en la 
Provincia de Buenos Aires. El problema central de nuestra investigación es el estudio de las características lingüísticas que presentan las escrituras de los/as niños/as en situación de contacto con el guaraní (bilingües guaraní-español/niños hispanohablantes en contacto con el guaraní) en los inicios de la alfabetización, y qué significados cognitivos y culturales se representan en esas opciones lingüísticas. Se propone, para ello, un trabajo de campo en una escuela del primer cordón del conurbano bonaerense a las que asisten niños y niñas que presentan contacto lingüístico del tipo aquí considerado. En este artículo plantearemos algunos principios teóricos, las resoluciones metodológicas adoptadas y las hipótesis planteadas hasta el momento.

Nuestra investigación se encuadra y sustenta en los principios de la teoría de la variación morfosintáctica propuestos por la Etnopragmática (García, 1995; Martínez, 2000, 2009). Si bien el contacto guaraní-español en el ámbito escolar se ha abordado desde otros marcos epistemológicos distintos al que presentamos aquí (Avellana y Brandani, 2020; Gaona Velázquez, 2012; Krasan et al., 2017), no es nuestra intención, por el momento, establecer un diálogo ni discutir con otras perspectivas. En este trabajo pretendemos explicitar el marco teórico metodológico de nuestra investigación, puesto que consideramos que esta perspectiva nos permite interpretar y explicar los fenómenos producto de las situaciones de contacto atendiendo a los principios cognitivos y comunicativos que rigen al uso del lenguaje, desde un punto de vista variacionista, a los que la Etnopragmática presta especial atención.

Dentro del marco teórico-metodológico en el que se inserta esta investigación existen trabajos que estudian fenómenos de contacto tanto de la oralidad como en la escritura, principalmente en las producciones discursivas de estudiantes de Nivel Medio y Superior (Álvarez Garriga y Speranza, 2012; Arnoux y Martínez, 2000; Fernández, 2008, 2010; Lucas, 2007; Martínez 2015b; Martínez y Speranza, 2004; Martínez, Speranza y Fernández, 2006b, 2009; Speranza, 2003, 2004, 2014; Speranza et al., 2012; Speranza, Fernández y Pagliaro, 2012).

El conocimiento sobre lo que sucede con las marcas del contacto en las escrituras de niños en el Nivel Primario del conurbano bonaerense, por tanto, junto con la explicación e interpretación de esos fenómenos destinado a docentes del nivel, es casi inexistente. No hay producciones que generen conocimiento sobre lo que sucede en ese nivel educativo, ni materiales destinados a docentes que tengan afán no solo descriptivo sino, sobre todo, analítico y explicativo.

Ante esta vacancia, nuestra investigación se propone generar conocimiento sobre los fenómenos de contacto guaraní-español en el Nivel Primario, específicamente aquellos presentes en las marcas que se encuentran en las prácticas de escritura de niños/as al comienzo de la alfabetización. Consideramos que es necesario y propicio abordar el uso 
variable del lenguaje que realicen los sujetos en la escritura aún -o precisamente por ello- bajo la presión normativa que presenta la producción escrita. Nos proponemos, con ello, generar conocimiento que aporte para la reflexión docente en torno a las lenguas que usan los alumnos, las características que el contacto lingüístico presenta y el modo en que este se expresa en los usos gramaticales que emergen en el español. Asimismo, pretendemos generar conciencia en torno a la coexistencia de distintas variedades no estándares del español en el aula, y la incidencia que ese reconocimiento puede tener en la enseñanza, específicamente, de la escritura.

\section{Un marco teórico interpretativo de la variación: la Etnopragmática}

Tal como hemos mencionado en la introducción, nuestra investigación se encuadra en los principios de la teoría de la variación morfosintáctica según los postulados teóricos de la Etnopragmática (García, 1995; Martínez, 2000, 2009). El análisis etnopragmático se interesa por explicar los procesos cognitivos que subyacen a las elecciones gramaticales que realizan los usuarios de la lengua; por ello, considera central indagar el modo en que el desvío de la frecuencia relativa de uso de las formas revela perspectivas cognitivas (García, 1995). En este sentido, en el uso variable de las formas lingüísticas (especialmente de la morfosintaxis) cobra especial interés descubrir qué contexto pragmático favorece qué forma/s lingüística/s, puesto que son los contextos de aparición los que podrán explicar la presencia de aquellas (García, 1995).

La Etnopragmática se interesa tanto por los principios cognitivos subyacentes a las elecciones lingüísticas que los sujetos hacen como por los aspectos comunicativos que motivan esas elecciones; desde esta perspectiva, la variación morfosintáctica se justifica por/en las necesidades comunicativas de los sujetos, y se vincula con procesos cognitivos que se encuentran implícitos en el uso del lenguaje. Se opone, por tanto, a la existencia de formas y estructuras lingüísticas preexistentes al uso del lenguaje: concibe a la gramática y a la estructura morfosintáctica de las lenguas no como algo dado $a$ priori sino como emergente, producto del uso que los sujetos hacen del lenguaje, en función de su perspectiva de mundo y de sus necesidades comunicativas. Las formas lingüísticas ocurren dónde y cómo lo hacen porque "son señales significativas usadas por seres inteligentes con el fin de transmitir mensajes" (Martínez, 2009, p.204).

Esta perspectiva sostiene que las formas morfosintácticas alternantes no expresan el mismo contenido semántico: por el contrario, se considera que distintas opciones representan manifestaciones de las perspectivas culturales y cognitivas de los sujetos usuarios del lenguaje. Los usos alternantes no implican, tal como se sostiene en el análisis sociolingüístico de tradición laboviana, decir lo mismo de maneras diferentes (Labov, 1983): el hecho de que dos o más formas lingüísticas se encuentran en variación 
implica que un evento puede representarse lingüísticamente desde perspectivas distintas, lo que supone que dos o más términos no son referencialmente iguales sino referencialmente equivalentes (Martínez, 2000); así, opciones o estrategias lingüísticas distintas reflejan perspectivas cognitivas distintas y expresan necesidades comunicativas distintas (Martínez, 2000; Speranza, 2010, 2014).

Como hemos visto hasta aquí, esta perspectiva de análisis se propone explicar los procesos cognitivos por los cuales los sujetos resuelven sus necesidades comunicativas en relación con las perspectivas culturales que representan. Por lo tanto podemos decir que el análisis etnopragmático es una perspectiva discursiva que parte de la consideración de que "las combinaciones sintácticas y su evidente regularidad son motivadas por principios cognitivos generales a los que el hablante apela para lograr relevancia y coherencia comunicativas" (Martínez, 2009, p.269) y, por ello, ha focalizado su interés en mostrar cómo la frecuencia de uso de las formas lingüísticas "constituye un síntoma de la perspectiva cognitiva del hablante y refleja pautas culturales" (p. 269).

En este punto se puede entender cómo esta perspectiva resulta propicia para abordar el estudio de situaciones de contacto de lenguas como la que abordamos en nuestra investigación. Muchos trabajos muestran la pertinencia de este enfoque teórico para el análisis de situaciones de contacto lingüístico, puesto que permite descubrir las estrategias a través de las cuales los sujetos dan cuenta de su visión del mundo en el uso del lenguaje, y cómo este se evidencia en el contacto (Álvarez Garriga y Speranza, 2012; Martínez, 2000, 2003, 2004, 2006, 2010a, 2010b, 2012, 2013, 2015a; Martínez y Bravo de Laguna, 2018; Martínez y Speranza, 2009a, 2013; Martínez, Speranza y Fernández, 2006a, 2006c, 2010; Speranza, 2010, 2012b, 2013, 2014, 2015, entre otros). Tal como señala Angelita Martínez (2009), el enfoque etnopragmático permite analizar fenómenos de variación lingüística, puesto que proporciona "herramientas metodológicas adecuadas para el estudio del uso variable de las formas lingüísticas" (p.259). Sus principios resultan particularmente útiles e iluminadores para el análisis de los procesos que subyacen a los fenómenos de bilingüismo y contacto lingüístico, atendiendo al hecho de que en el contacto de lenguas subyace siempre un "choque entre visiones de mundo y conceptualizaciones distintas [...]. Se produce una tensión entre culturas y cosmovisiones que entran en contacto, con el consecuente trasvase de una en otra" (Martínez, 2009, p.269).

Siguiendo los planteos del marco teórico adoptado, consideramos que es en el análisis de la morfosintaxis donde es posible ver en los usos alternantes las distinciones semánticas que estos implican desde el punto de vista cognitivo, cultural y comunicativo, pues en esta se hacen presentes las marcas del guaraní que, en este caso, se trasvasan al español. Dentro de este marco teórico, las diferencias que aparezcan en las conceptualizaciones de los niños en las estructuras sintácticas utilizadas en la escritura pueden y deben ser 
interpretadas y explicadas en función de aspectos cognitivos, culturales y comunicativos, para poder ser consideradas en el proceso de enseñanza de la lengua escrita. Desde esta perspectiva teórica es posible, entonces, interpretar los fenómenos de contacto que se hacen presentes en las elecciones morfosintácticas que los niños de escuela primaria hacen en sus escrituras, yendo más allá de la descripción y permitiendo realizar interpretaciones de aquellos fenómenos de variación lingüística que la normativa ha tradicionalmente considerado 'errores'.

\section{El Problema}

El problema central de nuestro trabajo radica en conocer, analizar e interpretar las características que presentan las escrituras de los niños que se encuentran en situación de contacto con el guaraní en los inicios de la alfabetización, y cuya variedad del español se aleje de la estandarizada. En esa indagación, y siguiendo los principios planteados por la Etnopragmática, nuestro propósito central es conocer qué significados cognitivos y culturales se representan en las opciones lingüísticas que los sujetos realicen.

Nuestra hipótesis inicial es que los usos que realicen los niños en situación de contacto del español escrito en los comienzos de la alfabetización presentarán, por un lado, algunas características similares a las de los niños monolingües y otras diferenciales; y que estas últimas representarán conceptualizaciones cognitivas provenientes de características culturales trasvasadas del guaraní. En ese sentido, a la luz de los trabajos realizados sobre algunos casos específicos de variación, planteamos que el uso del lenguaje que realizan los individuos en situación de contacto lingüístico "responde a la transferencia de conceptualizaciones propias de la lengua de contacto" (Speranza et al., 2012), y que será la frecuencia relativa de uso de esas opciones la que nos permitirá conocer si se trata de estrategias etnopragmáticas (García, 1995). La presencia del contacto lingüístico se evidencia en las características que presentan las distintas variedades de una misma lengua, características que constituyen "procesos de transferencia por los cuales se pone de manifiesto el perfilamiento cognitivo que el individuo adopta de la escena representada" (Speranza, 2010, p.93), para lo cual los sujetos utilizan creativamente las posibilidades gramaticales del español.

Dicho lo anterior, planteamos que las elecciones que realicen los niños en situación de contacto representarán diferencias con las opciones sintácticas que hagan quienes presenten una variedad del español más cercana a la estandarizada, en función de conceptualizaciones cognitivas que responden a diferencias culturales y a las características de la lengua de sustrato en cuestión. El análisis propuesto será posible solamente si se realizan comparaciones con niños monolingües sin contacto con lenguas indígenas, de modo de poder apreciar cuáles de las manifestaciones son idiosincrásicas 
del contacto con el guaraní y cuáles son usos más 'generales' que pueden encontrarse también en hablantes y escribientes sin contacto. A partir de esas comparaciones, realizaremos análisis e interpretaciones de aquellos aspectos que tradicionalmente se han considerado usos alejados de la norma lingüística y de la variedad estandarizada del español oral y escrito, focalizando en este caso en sus escrituras. Indagaremos en las conceptualizaciones subyacentes a las manifestaciones lingüísticas, en función de las relaciones existentes entre la lengua española y la lengua guaraní.

En cuanto a la materialidad con la que trabajaremos, es importante señalar que hemos decidido estudiar producciones lingüísticas escritas y no orales puesto que consideramos necesario indagar qué sucede con la variación lingüística en la escritura. La escritura, en general, ha sido excluida de los estudios de variación lingüística; sin embargo, desde el marco epistemológico de la Etnopragmática, consideramos que en las manifestaciones escritas se ponen también de manifiesto las particularidades del contacto lingüístico. En el relevamiento que hemos realizado hasta el momento respecto de los trabajos existentes, encontramos una vacancia respecto de los estudios sobre variación lingüística en las escrituras de niños de Educación Primaria. Es necesario focalizar en la investigación en la escritura, en tanto en la escuela se lo suele considerar una práctica -por su naturalezamás ligada a la norma, a la corrección lingüística, a la prescripción. Consideramos que es necesario y propicio abordar el uso variable del lenguaje que realicen los sujetos en la escritura aún -o precisamente por ello- bajo la presión normativa que presenta la producción escrita. Al mismo tiempo, nos permite pensar en las relaciones que se establecen entre oralidad y escritura en el ámbito educativo, y las concepciones que se tienen en torno a la escritura y la variación dentro de esta.

Puesto que nuestra investigación se encuentra aún en sus etapas iniciales, en principio nos proponemos indagar -en las producciones escritas que relevemos- fenómenos lingüísticos presentes en el español en contacto con el guaraní que han sido documentados tanto en trabajos previos dentro de la Etnopragmática (Lucas, 2007; Arnoux y Martínez, 2000a, 2000b; Martínez, 2000, 2003, 2009, 2010b, 2010c, 2013, 2015a; Speranza, 2010, 2012b, 2013, 2014; entre otros) como en trabajos de otras perspectivas teóricas (Abadía de Quant, 1996; De Granda, 1995; González Sandoval, 2005; Krivoshein de Canese, 1995; Krivoshein y Corvalán, 1983; Palacios, 1998, 2019; entre otros). Considerando estos antecedentes en las producciones escritas que relevemos inicialmente focalizaremos el análisis en los siguientes aspectos; los ejemplos han sido tomados de González Sandoval (2005) y Lucas (2007):

- usos alternantes de las preposiciones locativas en/a/por junto con verbos que indican desplazamiento (ejemplo: ir en casa vs. ir a casa; tenía una hamaca colgada por un árbol vs. tenía una hamaca colgada en un árbol); 
- usos alternantes de pronombres clíticos lo/la/le (ejemplo: le miró vs. lo miró/la miró; le ayudo vs lo/la ayudo);

- alternancia en la concordancia sintáctica en género (ejemplo: una mapa linda; estuvo lindo la visita; estaban muy ricos las torta);

- alternancia en la concordancia sintáctica en número (ejemplo: mis compañero; en el paisaje había mucho arbole; llegó los profesore; comenzó las vacaciones);

- omisión de artículos y determinantes (ejemplo: té para dolor de cabeza)

- utilización de tiempos verbales en el discurso como estrategia para expresar la evidencialidad, y

- omisión del pronombre personal objetivo de tercera persona (ejemplo: uno se asustan cuando ve).

Planteamos que en las producciones escritas que relevemos las características que difieren del español estandarizado presentarán una alta frecuencia, y que es posible que esa frecuencia sea aún más alta al tratarse de niños que se encuentran en los inicios de las prácticas de escritura. Tal como sostienen Arnoux y Martínez (2000b), el dominio que cada individuo tiene de la lengua escrita está en relación con las prácticas de escritura previas; por lo tanto, el hecho de que se trate de escritores 'novatos' podría ejercer una influencia en la frecuencia de aparición de las formas provenientes del contacto, en tanto la distinción entre oralidad y escritura no esté todavía establecida, y las prácticas de escritura pueden estar aún muy permeadas por la oralidad.

Es importante señalar que parte importante del problema planteado surge del hecho de que la presión normativa no siempre puede 'resolver' o 'corregir' aquellas formas alternantes que responden a conceptualizaciones cognitivo-culturales. Esto último, en la práctica educativa, genera un conflicto entre esas manifestaciones y las prácticas de escritura que la escuela espera y desea; en ese choque reside la importancia de las interpretaciones y explicaciones que nuestro trabajo pueda aportar para elaborar, a partir de allí, una mirada que articule con la enseñanza de la escritura.

\section{Metodología: algunas resoluciones}

Las resoluciones metodológicas adoptadas se han hecho siguiendo principios teóricos y metodológicos de la Etnopragmática, y atendiendo a la necesidad de configurar un cuadro sociolingüístico lo más claro posible de la población de la que se trabajará. Se realizará un estudio de caso en una escuela del Partido de Lomas de Zamora a la que asisten niños provenientes de Paraguay y de provincias del Noreste argentino (Formosa, Misiones, Corrientes, Chaco). La selección de la escuela se ha hecho a partir del conocimiento -por indagaciones previas- que gran parte de la población de niños y niñas 
que asisten a esta se encuentran en algún grado de contacto con el guaraní. La elección de una sola escuela se funda en el hecho de que el tipo de acercamiento propuesto será de tipo etnográfico (Rockwell, 2009) y requerirá trabajo intensivo y participativo de toda la comunidad (docentes, directivos, estudiantes).

A partir de esa aproximación, se realizará una caracterización antropológica y sociolingüística. El enfoque metodológico comprenderá la implementación de distintos instrumentos para recolectar datos: la selección de la muestra se realizará utilizando técnicas tomadas de la Antropología Social y la Etnografía (Geertz, 1983; Guber, 2001; Rockwell, 2009) y la Sociolingüística (Hernández Campoy y Almeida, 2005; Moreno Fernández, 1990; Speranza, 2012a). La metodología de análisis adoptada se enmarca en los postulados de la Etnopragmática (García, 1995; Martínez, 2000, 2009; Martínez y Speranza, 2009b; Martínez, Speranza y Fernández, 2009; Speranza, 2012a).

Algunas de las herramientas que se utilizarán para la recolección de datos serán:

- solicitud de datos censales que puedan brindar información sobre los lugares de procedencia de los niños y sus familias;

- encuestas directas del tipo cuestionario: se elaborará una encuesta siguiendo el modelo planteado por Speranza (2012a), adaptada a la realidad de la escuela en cuestión;

- entrevistas con directivos y docentes en las que se indagará sobre las características sociolingüísticas de los niños y se consultará acerca de las dificultades que encuentran al alfabetizar a los niños que están en/presentan algún tipo de contacto con el guaraní;

- conversaciones dirigidas con los niños en el contexto áulico para poder conocer -lo más ajustadamente posible- sus lugares de procedencia, si efectivamente presentan contacto con la lengua guaraní y cuál es el grado de bilingüismo que presentan (se utilizarán como complemento de las encuestas);

- observación participante;

- registros de campo, y

- relevamiento de producciones escritas de los estudiantes.

Es preciso señalar que en este trabajo adoptaremos una noción amplia de contacto lingüístico, entendido como un continuum que va desde niños bilingües en guaraní español -sea el grado que sea: hablantes, semihablantes o hablantes cuasipasivos, Dorian (1982)- hasta niños monolingües, pero cuya variedad de español están influenciada por el guaraní. Tal como señalan Martínez, Speranza y Fernández (2009), esta elección nos permitirá adoptar una noción de comunidad de habla que contemple "sus márgenes 
operativos" (Dorian, 1982) que resultará muy útil para analizar el desempeño de un amplio espectro de sujetos que -con diferentes grados de contacto con la lenguapresentan características que en la escuela se tornan 'conflictivas' para la enseñanza y el aprendizaje del español estándar, en general, y de la escritura en esa variedad, en particular.

Al seguir los planteos teóricos y metodológicos de la Etnopragmática, y puesto que una parte importante del análisis de esta perspectiva es no solo cualitativa sino también cuantitativo -en tanto sostiene como necesario indagar si existen regularidades en las ocurrencias lingüísticas usos alejados de la norma lingüística y de la variedad estandarizada-, el tipo de análisis de los datos será de tipo cuali y cuantitativo. Esto implicará hacer un relevamiento de las características que, por trabajos anteriores, sabemos existentes en el contacto del español con el guaraní junto con otras que puedan surgir en el análisis concreto del corpus; implicará, también, reconocer sus contextos discursivos de aparición y realizar análisis estadísticos de esas ocurrencias en contexto. El análisis cuantitativo será de tipo estadístico; se llevará a cabo bajo la premisa de que la frecuencia relativa de las formas es sensible al contexto, y que la frecuencia de uso de una forma lingüística en un contexto determinado depende de la congruencia entre el significado de la forma y su contexto de aparición (García, 1995).

\subsection{Población seleccionada}

Tal como se señaló, se realizará un estudio de caso en una escuela del Partido de Lomas de Zamora a la que asisten niños provenientes de Paraguay y de provincias del Noreste argentino que presentan distintos grados de contacto con la lengua guaraní. Durante el trabajo de campo se relevarán escrituras de niños de entre tercero y quinto grado. La elección de las edades y los grados ha sido pensada en función del nivel de escritura que los niños manejen, pues es preciso que escriban por sí mismos, al menos narrativas breves, ya que el análisis propuesto se hará sobre la producción de textos reales. Esta decisión se sostiene en el hecho de que uno de los propósitos de la Etnopragmática consiste en estudiar el comportamiento de las formas lingüísticas en contexto (es decir, cuál es el aporte que los signos hacen al discurso); no se analizan formas lingüísticas aisladas, oraciones descontextualizadas ni emisiones ad hoc: se analizan los contextos en los que las formas lingüísticas ocurren y cuál es la frecuencia con que aparecen; por lo tanto importa qué formas aparecen, en dónde (contexto) y cuánto (frecuencia de uso). Esto último resulta especialmente relevante, puesto que se considera que la frecuencia de uso de de las formas lingüísticas en el discurso representan estrategias etnopragmáticas por parte de los usuarios (García, 1995). 
A partir del diagnóstico realizado, se diseñarán dos situaciones didácticas con los docentes de los grados seleccionados, que requerirán por parte de los niños la producción de dos tipos de narrativas breves: una narrativa personal, en la que se relate algún suceso (personal, familiar, comunitario) conocido y vivido por ellos; otra narrativa, en la que se renarre un relato leído o narrado por los docentes, u otro tipo de narración producto del/ los trabajo/s propuesto/s por los docentes. La selección de los tipos textuales está guiada por la alta frecuencia que suelen tener en el contexto áulico, sobre todo en los primeros años de la escolaridad; se los selecciona, entonces, por la familiaridad que presentan, para los alumnos, los textos narrativos, y por la cercanía que genera la narración de eventos y relatos conocidos por los niños, tanto personalmente como de/en su entorno sociocomunitario; por ello, consideramos que serían especialmente permeables a la aparición de aspectos que responden a conceptualizaciones cognitivo-culturales.

La recolección de datos se hará en dos etapas: en la primera, se relevarán escrituras sin intervención ni de la investigadora ni de la/s docente/s a cargo de los grupos, como modo de poder hacer un diagnóstico. En una segunda etapa, se elaborarán propuestas de escritura en el aula (conjuntamente con la/s docente/s, a partir de textos que los/as niños/as acostumbren a escribir) que eliciten escrituras de los/as niños/as participantes de la investigación. Serán estas producciones las que -enmarcadas en una propuesta pedagógica concreta- serán analizadas para conformar el corpus de la investigación.

Puesto que la perspectiva teórica adoptada considera importante la 'frecuencia de uso' como reflejo de estrategias etnopragmáticas (García, 1995), se plantea como necesario contrastar las escrituras relevadas con otras de sujetos que no presenten contacto lingüístico como el que se indagará, pues ese contraste nos permitirá saber qué aspectos qué características son idiosincrásicas del contacto lingüístico y cuáles pertenecen a niños/as que se encuentran aún en proceso de aprendizaje de la escritura y de construcción de aspectos normativos de la escritura. A este efecto, se seleccionará otra escuela en se tenga conocimiento de que sus estudiantes, mayoritariamente, no presentan contacto con otras lenguas. En el 'grupo control', los grados seleccionados y los tipos de escrituras relevadas serán del mismo tipo que en las del grupo sobre el cual hace foco la investigación, de modo que los datos obtenidos puedan ser comparables. El análisis que se llevará a cabo sobre ese corpus será, también, de tipo cualitativo y cuantitativo.

\section{Aportes potenciales o sentido del conocimiento a construir}

Nuestra investigación pretende generar conocimiento lingüístico que pueda relacionarse, a futuro, con propuestas didácticas para la enseñanza de la escritura en contextos multiculturales en el Nivel Primario. Tal como hemos señalado, poco se sabe 
respecto de la variación lingüística producto del contacto del español con el guaraní en escuelas primarias del AMBA, específicamente en las prácticas de escritura. Por este motivo, nuestro trabajo pretende generar conocimiento que no implique solamente descripción de los fenómenos de contacto, sino que, fundamentalmente, explique cómo y por qué se producen esos usos lingüísticos alternantes respecto de los considerados normativamente correctos. Consideramos que el hecho de realizar descripción y explicación de los usos alternantes en las producciones escritas nos permitirá brindarles a los docentes conocimientos para comprender a la variación como parte integrante e inseparable de todas las manifestaciones lingüísticas de los sujetos, no solamente de/en la oralidad.

El tipo de investigación propuesta pretende mostrar que la variación no es azarosa sino sistemática, incluso en la escritura, y que en tanto represente aspectos comunicativos, cognitivos y culturales no puede ser simplemente corregida ni borrada bajo el peso de las normas de la variedad estandarizada del español. Puesto que consideramos que es posible que en las producciones escritas relevadas, las características que difieren del español estandarizado presenten una frecuencia mucho más alta respecto de la documentada en trabajos previos dentro de la misma línea teórica -pues los niños se encuentran en proceso de aprendizaje de las prácticas de escritura y de los aspectos lingüísticos asociados- esperamos a partir de ello aportar conocimiento para pensar en la alfabetización en contextos de diversidad lingüística desde los inicios de la escolaridad.

Junto con ello, los resultados que obtengamos de las comparaciones realizadas en producciones de sujetos que no presenten contacto lingüístico del tipo investigado en este trabajo nos permitirán aportar conocimiento sobre qué características son idiosincrásicas de ese contacto, y cuáles son propias de sujetos que se encuentran aún en proceso de aprendizaje de la escritura y de construcción de sus aspectos normativos; consideramos que ello podrá contribuir a la enseñanza de la escritura tanto en contextos en donde haya contacto lingüístico como en aquellos en los que ese contacto no esté presente.

El conocimiento en torno a la variación lingüística situada pretende generar, a futuro, la posibilidad de pensar en acciones que integren a la variación en la enseñanza tanto de la gramática como de la escritura. La dimensión interpretativa de esta investigación pretende aportar también, a futuro, para la elaboración de materiales y propuestas concretas que puedan desplegarse en las aulas, al tiempo que puedan también formar parte de distintas instancias en la formación docente para el Nivel Primario.

La noción amplia de comunidad de habla considerada en este trabajo permitirá reconocer que las características identificadas no corresponden solo a sujetos hablantes de guaraní (es decir, a quienes prototípicamente se considera bilingües) sino que estas 
formas de la diversidad lingüística pueden aparecer en manifestaciones de sujetos que son monolingües en español. De ese modo, pretenderemos brindar herramientas que permitan pensar a la variación como una noción fundamental para la enseñanza y el aprendizaje de las prácticas de oralidad y escritura en la escuela, atendiendo al hecho de que los fenómenos de variación lingüística forman parte indisoluble de los usos que los sujetos hacen del lenguaje y que, por ello, deben ser considerados en el proceso de enseñanza. Asimismo, pretendemos aportar a la construcción de concepciones no estigmatizantes de la variación lingüística, que aporten a una mirada integradora de la variación lingüística y cultural en la alfabetización.

Finalmente, consideramos que los resultados de esta investigación resultarán de suma importancia para generar conocimiento sobre la diversidad lingüística y cultural en el Nivel Primario -momento central en el proceso de alfabetización- que aporte a la comprensión del bilingüismo, el contacto de lenguas y la diversidad lingüística como inseparables de la realidad cognitiva y cultural de los sujetos usuarios del lenguaje y, por ello, imprescindibles para el aprendizaje y la enseñanza de la escritura.

\section{REFERENCIAS BIBLIOGRÁFICAS}

Abadía de Quant, I. (2006). Guaraní y español. Dos lenguas en contacto en el nordeste argentino. Signo y seña, 6, 201-233.

Álvarez Garriga, D. y Speranza, A. (2012, 7 y 8 de noviembre). La incidencia de la variación gramatical en la enseñanza de lenguas [ponencia]. IV Jornadas de Español como Lengua segunda y Extranjera, La Plata, Argentina. http://www.memoria.fahce.unlp. edu.ar/trab_eventos/ev.2731/ev.2731.pd

Arnoux, E. y Martínez, A. (2000a). Las huellas del contacto lingüístico. Su importancia para una didáctica de la escritura. En M. C. Rébola y M. C. Stoppa (Ed.), Temas actuales en Didáctica de la Lengua. Centro de Lingüística Aplicada. Facultad de Humanidades y Artes. Universidad Nacional de Rosario.

Arnoux, E. y Martínez, A. (2000b, 18 al 20 de mayo). Del oído al ojo: la variación en la producción escrita[ponencia]. Congreso de la Asociación Lingüística y Filológica de América Latina, Groningen, Holanda.

Avellana, A. y Brandani, L. (2020). La adquisición del lenguaje en contextos multilingües. Aportes para la reflexión sobre el contacto de lenguas español-guaraníy su proyección en el ámbito escolar. En Brandani, L.; Califa, M.; Magno, C. (eds) La adquisición del lenguaje y la enseñanza de segundas lenguas II. Universidad Nacional de General Sarmiento. pp. 15-40

Censabella, M. (1999). Las lenguas indígenas de la Argentina. Una mirada actual. EUDEBA. 
De Granda, G. (1995). Un proceso bidireccional de transferencia lingüística por contacto. Boletín de Filología, 35(1), 163-177. https://boletinfilologia.uchile.cl/index.php/ BDF/article/view/19204/20327

Dorian, N. (1982). Defining the speech community to include its working margins. En S. Romaine (Ed.), Sociolinguistic variation in speech communities (pp. 24-35). Edward Arnold.

Fernández, G. (2008). Interculturalidad en la escuela media: Ia alternancia preposicional como estrategia discursiva en sujetos en situación de contacto quichua/quechuaespañol. [Tesis de maestría inédita]. Buenos Aires, Instituto de Educación Superior "Dr. Joaquín V. González".

Fernández, G. (2010). El desafío de la escritura en el aula multicultural: una propuesta didáctica \{ponencia]. Actas del Congreso Regional de la Cátedra UNESCO en Lectura y Escritura. Cultura Escrita y Políticas Pedagógicas en las Sociedades Latinoamericanas Actuales. Buenos Aires, Universidad Nacional de General Sarmiento. https://www. ungs.edu.ar/wp-content/uploads/2012/11/Libro-Actas.pdf

García, E. (1995). Frecuencia (relativa) de uso como síntoma de estrategias etnopragmáticas. En K. Zimmermann (Ed.). Lenguas en contacto en Hispanoamérica (pp. 51-72). Vervuert, Ibero-Americana.

Gaona Velázquez, I. (2012). El bilingüismo guaraní castellano y su incidencia en la producción escrita de los alumnos al final del primer ciclo de la EEB [Tesis de Maestría, Universidad Nacional de La Plata]. Archivo digital. http://www.memoria.fahce.unlp. edu.ar/tesis/te.834/te.834.pdf

Geertz, C. (1983). Descripción densa. Hacia una una teoría interpretativa de la cultura. En C. Geetz, La interpretación de las culturas (pp ). Gedisa.

González Sandoval, G. (2005). Las transferencias del guaraní en escuelas rurales de Corrientes. Hacia una didáctica del contacto de lenguas. Universidad Nacional de Rosario.

Guber, R. (2001). La etnografía. Método, campo y reflexividad. Norma.

Hernández Campoy, J. y Almeida, M. (2005). Metodología de la investigación sociolingüística. Comares.

Krasan, M. et. al. 2017. Material de consulta para el docente en contextos de diversidad lingüística. Estructuras constrastivas guaraní-español/quechua-español. Editorial de la Facultad de Filosofía y Letras, Universidad de Buenos Aires. http://publicaciones. filo.uba.ar/sites/publicaciones.filo.uba.ar/files/Material de consulta para el docente en contextos de diversidad lingüística_interactivo_0.pdf- 
Krivoshein de Canese, N. (1995). El guaraní como lengua aglutinante y polisintética. Nemity, 31.

Krivoshein de Canese, N. y Corvalán, G. (1983). El español del Paraguay en contacto con el guarani. Centro Paraguayo de Estudios Sociológicos.

Labov, W. (1983). Modelos sociolingüísticos. Cátedra.

Lucas, M. (2007). Rasgos del aymara y del guaraní en el español de alumnos en situación de contacto lingüístico. Signo y Seña, 17, 247-267. http://revistascientificas.filo.uba. ar/index.php/sys/article/view/5756/5145

Martínez, A. (2000). La Sociolingüística desde el enfoque etnopragmático. En: Arnoux, E.; Lagorino, C; Romero, D.; Rubione, A. (eds.) Lenguajes: Teorías y Prácticas. (pp. 8594). Primer simposio de la Maestría en Ciencias del Lenguaje. Instituto Superior del Profesorado "Joaquín V. González". Buenos Aires: Gobierno de la Ciudad de Buenos Aires. Secretaría de Educación.

Martínez, A. (2003). Etnopragmática. El castellano en contacto con lenguas aborígenes. En A. Palacios y A. García (Eds.) El indigenismo americano. Cuadernos de Filología, XLVIII (pp. 83-100). Universitat de Valencia.

Martínez, A. (2004). Variación lingüística y estrategias discursivas. En R. Kirsner; E. ContiniMorava y B. Rodríguez-Bachiller (Eds.), Cognitive and Communicative Approaches to Linguistic Annalysis (361-379). John Benjamins.

Martínez, A. (2006). "Lenguas en contacto: gramaticalización y frecuencia de uso." En: Actas del encuentro de Lenguas Indígenas Americanas. La Pampa: Universidad Nacional de La Pampa/Subsecretaría de Gobierno de La Pampa. 1-24.

Martínez, A. (2009). "Metodología de la investigación lingüística: el enfoque etnopragmático" En: Narvaja de Arnoux, Elvira (Directora), Escritura y producción de conocimiento en las carreras de posgrado. Bs. As., Santiago Arcos editor. 259-286.

Martínez, A. (2010a). Lenguas y variedades en contacto. Problemas teóricos y metodológicos. Revista Internacional de Lingüística Iberoamericana VIII, I (15), 9-31.

Martínez, A. (2010b). De España a América. Recategorización y desplazamientos en el sistema de clíticos". Olivar, 11 (14), 149-162. http://www.memoria.fahce.unlp.edu. ar/art_revistas/pr.4504/pr.4504.pdf

Martínez, A. (2010c, del 11 al 13 de agosto). Culturas, necesidades comunicativas y variación lingüística. 10 Congreso Regional de la Cátedra UNESCO en Lectura y Escritura: Cultura Escrita y Políticas Pedagógicas en las Sociedades Latinoamericanas Actuales, Ciudad Autónoma de Buenos Aires, Argentina. http://www.memoria. fahce.unlp.edu.ar/trab_eventos/ev.4965/ev.4965.pdf 
Martínez, A. (2012). El 'juego' en los sistemas gramaticales y la coexistencia de variedades de una lengua. Cuadernos de la ALFAL, 4, 212-122. http://www.mundoalfal.org/ sites/default/files/revista/04_cuaderno_010.pdf

Martínez, A. (2013). Variación pronominal en la Argentina: Los pronombres clíticos lo, la y le. En: L. Colantoni y C. Rodríguez Louro (Eds.). Perspectivas teóricas y experimentales sobre el español de la Argentina (pp. 397-416). Madrid: Iberoamericana.

Martínez, A. (2015a). ¿Cómo afecta la cultura a la gramática?: El caso de los clíticos en el español americano. Círculo de lingüística aplicada a la comunicación, 61, 186-210. http://www.memoria.fahce.unlp.edu.ar/art_revistas/pr.7088/pr.7088.pdf

Martínez, A. (Coord.) (2015b). Huellas teóricas en la práctica pedagógica: el dinamismo lingüístico en el aula Intercultural. Universidad Nacional de La Plata. http://www. memoria.fahce.unlp.edu.ar/libros/pm.387/pm.387.pdf

Martínez, A. y Bravo de Laguna, M. (2018, del 2 al 3 de mayo). El poder de la mirada y la mirada del poder: tensiones en el inter-juego de los lenguajes. I Jornadas Internacionales Agonalidad y Ritualidad. Freiburg, Alemania. http://www.memoria. fahce.unlp.edu.ar/trab_eventos/ev.13807/ev.13807.pdf

Martínez, A. y Speranza, A. (2004). El aporte de la etnopragmática a la práctica de aula en contextos de heterogeneidad lingüístico-cultural [ponencia]. I Congreso Internacional Educación, Lenguaje y Sociedad. Tensiones educativas en América Latina, La Pampa, Argentina.

Martínez, A. y Speranza, A. (2009a). Variaciones lingüísticas: usos alternantes. En E. Arnoux (Dir.), Pasajes (pp. 179 a 203). Biblos.

Martínez, A. y Speranza, A. (2009b). ¿Cómo analizar los fenómenos de contacto lingüístico?: Una propuesta para ver el árbol sin perder de vista el bosque. Lingüística, 21 (1), 87-107. http://www.memoria.fahce.unlp.edu.ar/art_revistas/pr.7089/ pr.7089.pdf

Martínez, A. y Speranza, A. (Eds.). (2013). Rumbos sociolingüísticos. Facultad de Filosofía y Letras, Universidad Nacional de Cuyo y Sociedad Argentina de Lingüística.http:// ffyl1.uncu.edu.ar/IMG/pdf/Martinez_y_Speranza_eds_2013..

Martínez, A.; Speranza, A. y Fernández, G. (2006a). Lenguas en contacto y perspectivas cognitivas: interculturalidad en Buenos Aires. UniverSOS, 3, 9-33. https://www. uv.es/ calvo/amerindias/numeros/n3.pdf

Martínez, A.; Speranza, A. y Fernández, G. (2006b). Diversidad lingüística y enseñanza de la lengua: hacia la concreción de una práctica educativa que contemple la realidad multicultural de la Ciudad de Buenos Aires y la Provincia de Buenos Aires. En A. 
Martinez (Coord.). El dinamismo lingüístico en el aula intercultural. Su aplicación en talleres para docentes.

Martínez, A.; Speranza, A. y Fernández, G. (2006c). Lenguas en contacto y perspectivas cognitivas: quechua-castellano en Buenos Aires. En J. Calvo Pérez (Ed.), Universos discursivos (pp. 9-33). Universidad de Valencia.

Martínez, A.; Speranza, A. y Fernández, G. (2009). El entramado de los lenguajes. La Crujía.

Moreno Fernández, F. (1990). Metodología sociolingüística. Gredos.

Palacios, A. (1998). Variación sintáctica en el sistema pronominal del español paraguayo: la elisión de pronombres objeto. Anuario de Lingüística Hispánica, XIV, 451-474. http://espanolcontacto.fe.uam.es/wordpress/wp-content/uploads/2017/02/ Variación-sintáctica-en-el-sistema-pronominal-del-español-paraguayo.pdf

Palacios, A. (2019). La reorganización de las preposiciones locativas 'a', 'en' y 'por' en el español en contacto con guaraní. Círculo De Lingüística Aplicada a La Comunicación, 78, 233-254. https://doi.org/10.5209/clac.64380

Rockwell, E. (2009). La experiencia etnográfica. Historia y cultura en los procesos educativos. Paidós.

Speranza, A. (2003). El desafío de la enseñanza de la lengua en contextos multilingües. Signos Universitarios Virtual, 2 (1). www.salvador.edu.ar/sv10-suvn.htm. Speranza, A. (2004, del 30 de marzo al 2 de abril). La variación lingüística en el uso correlativo de tiempos verbales: el caso del contacto quechua-castellano [ponencia]. I Congreso Internacional Políticas Culturales e Integración Regional. .

Speranza, A. (2010). Estrategias discursivas en la transmisión de la información: el español en contacto con lenguas americanas. Revista Internacional de Lingüística Iberoamericana VIII, 1(15), 89-115.

Speranza, A. (2012a). Comunidades de habla en el Gran Buenos Aires: una aproximación sociolingüística. En Identidades lingüísticas y culturales en contextos educativos. Imprex Ediciones.

Speranza, A. (2012b). Perspectivas culturales en la variación lingüística. Cuadernos de la ALFAL, 4, 126- 139. http://mundoalfal.org/es/content/cuadernos-de-la-alfal-no4

Speranza, A. (2013). Estrategias evidenciales en el español rioplatense: una aproximación desde la variación lingüística. En A. Martínez y A. Speranza (Eds.) Rumbos sociolingüísticos.. Volúmenes temáticos de la SAL. Facultad de Filosofía y Letras, UNCuyo. 
Speranza, A. (2014). La evidencialidad en el español americano. La expresión lingüística de la perspectiva del hablante. Iberoamericana.

Speranza, A. (2015). La construcción del punto de vista desde una perspectiva variacionista. En E. Narvaja de Arnoux y R. Bein (Eds.) Política lingüística y enseñanza de lenguas. Biblos.

Speranza, A. (Coord.); Fernández, G. y Pagliaro, M. (2012). Identidades lingüísticas y culturales en contextos educativos. Imprex Ediciones.

Speranza, A. et. al. (2012). Diversidad lingüística y cultural: hacia el desarrollo de prácticas inclusiva en la educación. En A. Molinari, Conocer para incidir sobre las prácticas pedagógicas (pp. 115 a 136). Ministerio de Educación de la Nación. http:// repositorio.educacion.gov.ar:8080/dspace/handle/123456789/109615

\footnotetext{
${ }^{1}$ Profesora y Licenciada en Letras por la Universidad de Buenos Aires (orientación Lingüística) y Especialista en Lectura, escritura y educación (FLACSO). Se desempeña como docente en los niveles Medio y Superior de la Provincia de Buenos Aires. Sus áreas de interés son la sociolingüística y las prácticas de enseñanza de la lectura y la escritura (incluyendo la alfabetización inicial) desde una perspectiva sociolingüística. Actualmente es maestranda en Escritura y Alfabetización en la Facultad de Humanidades y Ciencias de la Educación (UNLP).
} 